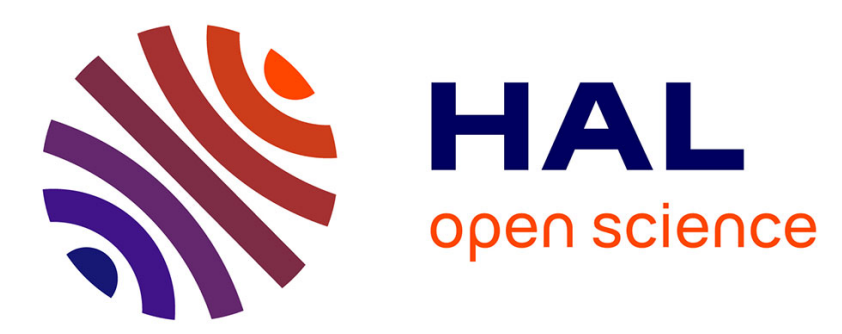

\title{
Control of nonlinear instabilities in Bessel beams using shaped longitudinal intensity profiles
}

\author{
Ismail Idrissi, Remo Giust, John Michaël Dudley, François Courvoisier
}

\section{To cite this version:}

Ismail Idrissi, Remo Giust, John Michaël Dudley, François Courvoisier. Control of nonlinear instabilities in Bessel beams using shaped longitudinal intensity profiles. Conference on Lasers and Electro-Optics, May 2017, San Jose, United States. hal-02134424

\section{HAL Id: hal-02134424 \\ https://hal.science/hal-02134424}

Submitted on 20 May 2019

HAL is a multi-disciplinary open access archive for the deposit and dissemination of scientific research documents, whether they are published or not. The documents may come from teaching and research institutions in France or abroad, or from public or private research centers.
L'archive ouverte pluridisciplinaire HAL, est destinée au dépôt et à la diffusion de documents scientifiques de niveau recherche, publiés ou non, émanant des établissements d'enseignement et de recherche français ou étrangers, des laboratoires publics ou privés. 


\title{
Control of nonlinear instabilities in Bessel beams using shaped longitudinal intensity profiles
}

\author{
I. Ouadghiri-Idrissi, R. Giust, J. M. Dudley, and F. Courvoisier \\ FEMTO-ST Institute, University of Bourgogne-Franche-Comté UMR-6174, 25030 Besancon, France \\ ismail.ouadghiri@femto-st.fr
}

\begin{abstract}
We show that tailored longitudinal intensity shaping of a non-diffracting Bessel beam can strongly reduce four wave mixing induced oscillations and stabilize nonlinear propagation at ablation-level intensities.

OCIS codes: (190.0190) Nonlinear optics; (140.3300) Laser beam shaping; (320.0320) Ultrafast optics.
\end{abstract}

Diffraction-free Bessel beams exploit conical energy flow to yield a near-uniform intensity distribution along a line focus. For high power laser pulses injected into dielectrics, this beam structure can sustain quasi propagationinvariant regimes of filamentation, which are highly advantageous in reducing nonlinear distortion and instabilities during propagation. However, residual nonlinear effects can still take place and induce significant oscillations of the on-axis intensity, which is detrimental in creating longitudinally-uniform structures in material processing [1].
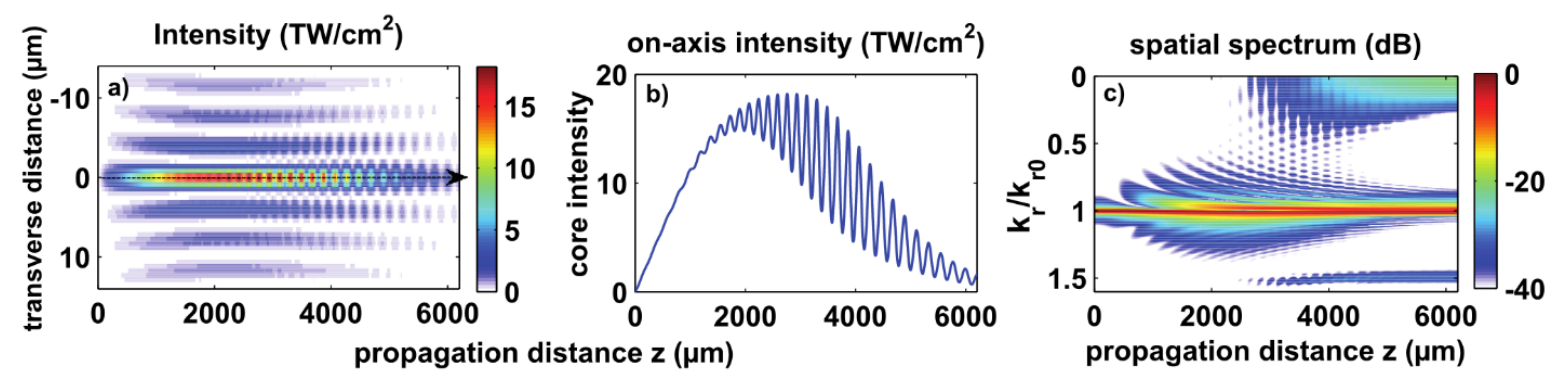

Fig. 1. a) Intensity distribution of a Bessel beam in the nonlinear regime, b) the corresponding on-axis intensity and c) the spatial spectrum distribution along the propagation distance. . Parameters of the simulations are: wavelength $\lambda=0.8 \mu \mathrm{m}$, linear refractive index $n=1.45$, nonlinear refractive index $n_{2}=2.4810^{-20} \mathrm{~m}^{2} / \mathrm{W}$, input energy: $E_{\text {in }}=6.6 \mu \mathrm{J}$, beam waist: $w_{0}=300 \mu \mathrm{m}$ and Bessel beam cone angle: $\theta=4^{\circ}$.

In this work, we show analytically and numerically that these intensity variations can be reduced through suitable shaping of the longitudinal intensity variation of the Bessel beam [2] injected into the dielectric. Figure 1 shows the problem we aim to solve. We plot results from numerical simulations of nonlinear propagation in fused silica of a Bessel-Gauss beam (generated e.g from an axicon) taking into account Kerr effect in a nonlinear Schrödinger equation model. Space-time effects and ionization are ignored in a first step. Figure 1(b) shows the profile of the onaxis intensity of the central core, where we see significant intensity modulation along $z$ due to the generation of new spatial frequencies from four wave mixing (FWM) $[1,3]$. The corresponding $z$-evolution of the spatial spectrum is shown in Fig. 1(c). After an initial stage of spectral broadening (propagation distance 0-2000 $\mu \mathrm{m}$ ), FWM between frequencies generated from the broadening pump around $k_{r 0}$ generates an axial wave $\left(k_{r} \sim 0\right)$ and a secondary Bessel beam $\left(k_{r} \sim \sqrt{2} k_{r 0}\right)$. Experimental signatures of this behavior have been reported in Ref. [3].

We use a recently developed flexible experimental approach to using a phase-only spatial light modulator to create Bessel beams with arbitrary longitudinal intensity profile at high intensity [2]. We have numerically modelled the nonlinear propagation of such arbitrary profiles, and considered three target intensity profiles as shown in Fig. 2(a) consisting of: a (usual) Bessel-Gauss profile (green dashed line) as in Fig. 1; a linear leading edge, constant intensity and parabolic decaying trailing edge (blue dashed line); a parabolic increase leading edge, constant intensity and parabolic decay trailing edge (red solid line).

The simulation results showing on-axis line profiles of the nonlinear evolution are shown in Fig. 2(b) and it is clear there is significant qualitative difference in the observed behavior. Specifically, the case of parabolic leading and trailing edges on either side of a zone of constant intensity (red curve) can be seen to lead to significantly reduced nonlinear oscillations. It is very apparent that the progressive intensity rise follows the target profile. Fig. 2(c) shows the evolution of the spatial spectrum with propagation distance. It illustrates clearly the large suppression of the additional spectral components around $k_{r} \sim \sqrt{2} k_{r 0}$ and $k_{r} \sim 0$ for the case of the parabolic intensity increase. 
a) target intensity profiles

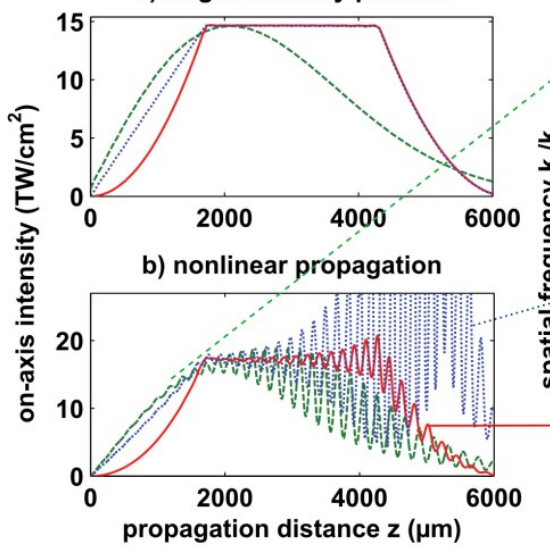

c) spatial spectra (dB)

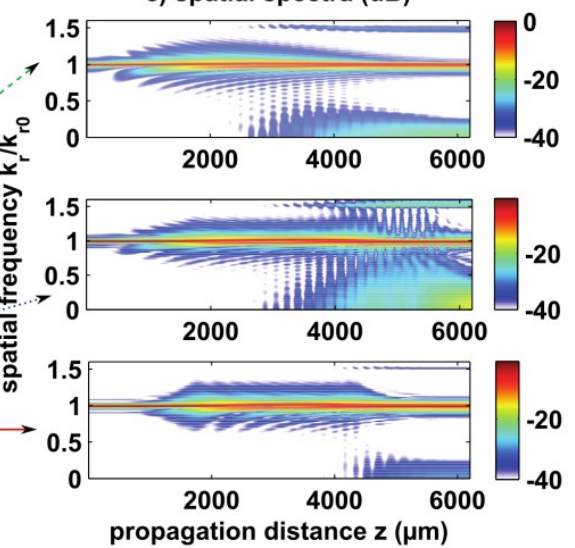

Fig 2. Simulation of the nonlinear propagation of three Bessel beams with different a) target on-axis intensity profiles. Evolution of their respective b) on-axis intensities and c) spatial spectra along propagation.

To theoretically explain these features, we have studied the generation of new spectral components through the four wave mixing process (FWM). We have analytically explained that the input spectral phase is a key parameter to explain the growth of novel spectral components by FWM. We plot the results of our analytical model in Fig. 3 below. The efficiency of the FWM process is much higher (Fig. 3(c), top) if the input beams have a quasi-flat spectral phase (Fig. 3(b), blue \& green), which is the case for the linear ramp and the Bessel Gauss profiles. In contrast, it is greatly reduced for the parabolic intensity growth (Fig. 3(c), bottom), where the input spatial spectrum has a rapidly varying phase evolving as a parabola (Fig. 3(b), red). The results of our reduced analytical model are in good agreement with the full numerical model of Fig. 2.
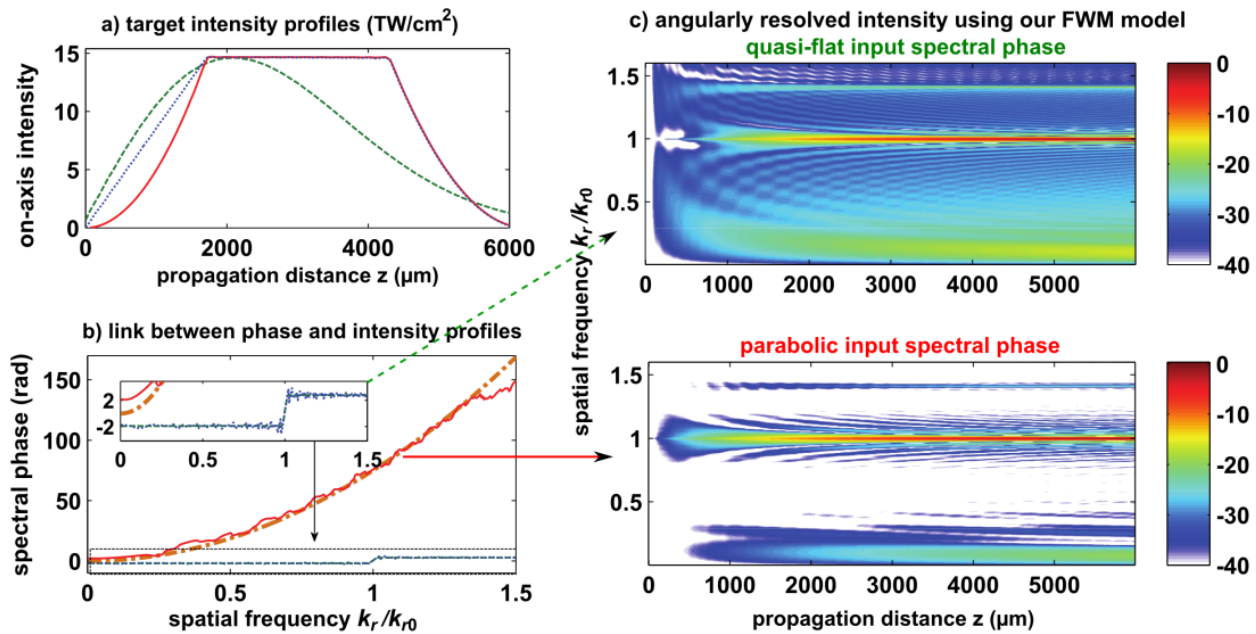

Fig 3. Semi-analytical results using our reduced FWM model: a) Target intensity profiles, b) corresponding input spectral phase distributions and c) the evolution of the spatial spectra (dB) along propagation in case of a quasi-flat (top) and parabolic (bottom) spectral phase distributions.

Additional time-resolved numerical simulations including nonlinear losses by multiphoton ionization also show comparable behavior. Our numerical results clearly show the potential of longitudinal beam shaping to control nonlinear intensity instabilities and open novel perspectives to control nonlinear propagation and filamentation.

We acknowledge funding from the European Research Council (ERC) under Horizon 2020 program (GA N ${ }^{\circ}$ 682032-PULSAR) in cooperation with Labex ACTION program,, contract ANR-11-LABX-0001-01.

\section{References}

[1] P. Polesana, M. Franco, A. Couairon, D. Faccio, and P. Di Trapani, "Filamentation in Kerr media from pulsed Bessel beams," Phys. Rev. A 77, 043814 (2008).

[2] I. Ouadghiri-Idrissi, R. Giust, L. Froehly, M. Jacquot, L. Furfaro, J. M. Dudley, and F. Courvoisier, "Arbitrary shaping of on-axis amplitude of femtosecond Bessel beams with a single phase-only spatial light modulator," Opt. Express 24, 11495-11504 (2016).

[3] R. Gadonas, V. Jarutis, R. Paskauskas, V. Smilgevicius, A. Stabinis, and V. Vaicaitis, "Self-action of Bessel beam in nonlinear medium," Opt. Commun. 196, 309-316 (2001). 